\title{
Prognostic Implications of Creatine Phosphokinase levels after Acute Myocardiaß Infarction-A Study of Low Risk Patients
}

\author{
Maj A Henderson* \\ BMedSci, MRCP, RAMC \\ Senior Specialist in Medicine, Cambridge Military Hos pital
}

SUMMARY: To determine the prognostic value of Creatine Phosphokinase (CPK) levels after acute myo cardial infarction in patients thought to be at low risk of death or major complications, 94 consecutive patients with definite myocardial infarction were divided into two groups according to peak CPK levels. Grous 5 I contained 23 patients with a peak CPK of less than 1000 units and Group II contained 71 patients. with a peak CPK level of greater than 1000. They were followed for six months or to death. A CPK of greater than 1000 was significantly associated with left ventricular failure, major arrhythmias and late death and seemed therefore to have value as an indicator of poor prognosis.

\section{Introduction}

Patients at high risk of major complications, early death or late death after myocardial infarction can be easily recognized. The adverse prognostic factors include previous myocardial infarction, age greater than 70 years, severe left ventricular failure, severe hypotension, cardiomegaly, bundle branch block, anterior wall infarction and very high levels of cardiac enzymes ${ }^{1,} 2,3$. Although patients with none of the above have on the whole a good outlook, providing they survive to reach hospital, some will die or develop a major complication. Identification of this subset of at risk patients has received little attention. Total Creatine Phosphokinase (CPK) release after infarction can be related to infarct size and to prognosis ${ }^{4}, 5,8,7$, but the methodology involved in the investigation is too complex and cumbersome to be of use in a working coronary care unit. This study has attempted to relate peak CPK level as measured on a daily basis in the manner common to most coronary care units to prognosis in patients thought to be at low risk of death or major complications following acute myocardial infarction.

\section{Method}

Inclusion Criteria: Patients thought to be at low risk from death or major complications admitted to the coronary care unit because of definite myocardial infarction were enrolled into the study. Definite myocardial infarction was defined as typical pain lasting for at least 30 minutes plus elevation of CPK to at least three times the upper limits of normal, plus sequential ST and $T$ wave changes of infarction or the development of $\mathrm{Q}$ waves of more than 30 milliseconds duration.

\footnotetext{
${ }^{*}$ Now at $B M H$, Dharan
}

Exclusion Criteria:

1 Age greater than 70 years

2 Insulin dependent diabetes

3 Patients taking Digoxin

4 Previous myocardial infarction

5 Right or left bundle branch block

6 Severe hypotension (systolic BP less than 90)

7 Severe pulmonary oedema

\section{Procedure}

Patients were assessed clinically on admission the coronary care unit and blood was taken imme्ध iately for CPK assay. Serial CPK levels were taken for the next three days. Serial ECGs were recor for at least the first three days and a chest $x$-ray was taken on admission and repeated there-after as indicated. Patients were managed in the usuap way except that IM injections, which were known to elevate CPK, were avoided. Patients were folo lowed to death or to six months from the day of admission. The following end points were noted:

1 Death

2 Ventricular fibrillation

3 Ventricular tachycardia (for the purpose of the study defined as aberrant ventricular com? plexes at a rate of greater than $120 /$ min lasting for longer than 30 seconds).

4 Supraventricular tachycardias (including atria fibrillation.

5 Left ventricular failure defined as chest $\mathrm{x}$-rayg evidence of pulmonary congestion plus a third heart sound, or chest x-ray evidence of pul으 monary oedema.

6 High grade atrio ventricular block requiring treatment.

7 Angina after discharge.

8 A further cardiac event requiring re-admis sion to coronary care. 


\begin{tabular}{|c|c|c|c|c|c|}
\hline & \multicolumn{2}{|c|}{ Group I } & \multicolumn{2}{|c|}{ Group II } & \multirow{2}{*}{$\begin{array}{c}\text { Standard } \\
\text { Error of } \\
\text { Difference } \\
\text { (SE) }\end{array}$} \\
\hline & $\begin{array}{c}\text { Number } \\
\text { (No) }\end{array}$ & $\begin{array}{c}\text { Percentage } \\
(\%)\end{array}$ & $\underset{\text { (No) }}{\text { Number }}$ & $\begin{array}{c}\text { Percentage } \\
(\%)\end{array}$ & \\
\hline Total & 23 & 100 & 71 & 100 & \\
\hline All Deaths & 2 & 9 & 9 & 13 & 7.2 \\
\hline Deaths before 7 days & 2 & 9 & 2 & 3 & 6.3 \\
\hline Deaths after 7 days & 0 & 0 & $\overline{7}$ & 10 & 3.6 \\
\hline Deaths after discharge & 0 & 0 & 4 & 6 & 2.8 \\
\hline LVF & 2 & 9 & 27 & 38 & 3.8 \\
\hline VF & 1 & 4 & 12 & 17 & 6.0 \\
\hline VT & 0 & 0 & 11 & 15 & 4.2 \\
\hline SVT & 0 & 0 & 14 & 20 & 4.8 \\
\hline Heart Block & 3 & 13 & 4 & 6 & 7.5 \\
\hline Angina after discharge & 6 & 26 & 23 & 32 & 10.7 \\
\hline Readmission to $\mathrm{CCU}$ & 4 & 17 & 9 & 13 & 8.8 \\
\hline
\end{tabular}

Abbr: LVF-Left Ventricular Failure. VF-Ventricular Fibrillation.

SVT-All supraventricular Tachyarrhythmias.

VT $=$ Ventricular Tachycardia

\section{Results}

Ninety-four consecutive patients with acute myocardial infarction without any major risk factors were enrolled into the study over a period of 12 months. They were divided into two groups. Group I with peak CPK levels of less than 1000 (23 patients) and Group II with peak CPK levels of more than 1000 (71 patients). The groups were closely similar with regard to age, sex, smoking habits, hypertension, angina, non insulin dependent diabetes and therapy with beta blockers or diuretics. The result of all patients are outlined in Table $I$. A total of 87 patients survived to leave hospital, of whom four subsequently died at home within the next six months. There was no significant difference in the rate of early death but deaths after seven days (Group I $-0 \%$ versus Group 2 $10 \%, \mathrm{SE} 3.6)$ and after discharge (Group I - $0 \%$ versus Group II $-6 \%$, SE 2.8) were significantly more common in Group II. Major arrhythmias were also significantly more frequent in Group II (Group I $17 \%$ versus Group II $58 \%$, SE 9.8). Left ventricular failure was significantly more common in Group II (Group I - 9\% versus Group II - 38\%, SE 8.3). Subset analysis of patients with anterior and inferior infarction was undertaken. In anterior infarction (Table II) death, major arrhythmias and left ventricular failure remained closely associated with Group II patients. However, in patients with acute inferior infarction (Table III) the association with left ventricular failure and arrhythmias was lost, although that with late death and supraventricular tachycardias remained. To exclude the possibility of falsely high CPK levels in Group II secondary to DC cardioversion (ventricular fibrillation was much more common in this group) a second subset analysis excluding patients with ven- tricular fibrillation was undertaken (Table IV). Late death, major arrhythmias, other than ventriculare fibrillation and left ventricular failure remained $\overline{0}$

Table II Anterior Infarction

\begin{tabular}{lrrrrrr}
\hline & \multicolumn{3}{c}{ Group I } & \multicolumn{3}{c}{ Group II } \\
\cline { 2 - 5 } & No & $\%$ & No & $\%$ & \\
\hline Total & 11 & 100 & 33 & 100 & \\
All Deaths & 0 & 0 & 5 & 15 & 6.20 \\
Deaths after 7 days & 0 & 0 & 3 & 6 & 4.10 \\
LVF & 1 & 9 & 17 & 51 & 12.2 \\
VF & 0 & 0 & 7 & 21 & 7.10 \\
VT & 0 & 0 & 6 & 18 & 6.7 \\
SVT & 0 & 0 & 7 & 21 & 7.1 \\
\hline
\end{tabular}

Table III Inferior Infarction

\begin{tabular}{lrrrrrr}
\hline & \multicolumn{3}{c}{ Group I } & \multicolumn{3}{c}{ Group II } \\
\cline { 2 - 5 } & No & $\%$ & No & $\%$ & SE \\
\hline Total & 12 & 100 & 38 & 100 & \\
All Deaths & 2 & 17 & 4 & 10 & 12 \\
Deaths after 7 days & 0 & 0 & 4 & 10 & 5 \\
LVF & 1 & 8 & 10 & 26 & 10.5 \\
VF & 1 & 8 & 5 & 13 & 9.5 \\
VT & 0 & 0 & 5 & 13 & 9.5 \\
SVT & 0 & 0 & 7 & 18 & 6.2 \\
\hline
\end{tabular}

Table IV Excluding Patients with VF

\begin{tabular}{lrrrrrr}
\hline & \multicolumn{3}{c}{ Group I } & \multicolumn{3}{c}{ Group II } \\
\cline { 2 - 5 } & No & $\%$ & No & $\%$ & SE \\
\hline Total & 22 & 100 & 59 & 100 & \\
All Deaths & 2 & 9 & 7 & 12 & 7.4 \\
Deaths after 7 days & 0 & 0 & 7 & 12 & 4.2 \\
LVF & 1 & 4 & 19 & 32 & 7.4 \\
SVT & 0 & 0 & 17 & 29 & 5.9 \\
VT & 0 & 0 & 9 & 15 & 4.6 \\
\hline
\end{tabular}


strongly associated with CPK level of greater than 1000 suggesting the association was not artefactual.

\section{Discussion}

Patients with poor prognosis after acute myocardial infarction can be identified by a variety of clinical and investigative parameters. Previous infarction, severe left ventricular failure, severe hypotension, bundle branch block, cardiomegaly advanced stage and gross elevation of cardiac enzymes especially total CPK are all adverse prognostic factors. Within the remaining patients who had none of the above and therefore who appear to be in low risk group, is a subset who develop major complications and have an increased mortality. Identifying this subgroup has proved difficult and has received relatively little attention. This study has attempted to evaluate the prognostic implications of peak CPK levels which are readily available in working coronary care units. A CPK of more than 1000 following myocardial infarction in patients with no adverse prognostic factors on admission seems to be significantly associated with the development of major arrhythmias, late death and left ventricular failure. Subset analysis has confirmed that the relationship remains strong when considering patients with anterior myocardial infarction, but interestingly is less definite with inferior infarction. This may be because death and complications after anterior infarction relates fairly closely to the amount of left ventricular muscle damaged, whereas an inferior infarction, frequently the right ventricle, is also infarcted causing severe haemodynamic upset, yet because of its relatively small mass contributing little to total CPK release.

High levels of CPK failed to predict angina after discharge from hospital nor did it predict further cardiac events (except death) requiring readmission to hospital. Peak CPK probably crudely reflects infarct size so the association with left ventricular failure, death and supraventricular arrhythmias, which are particularly common in patients with elevated left ventricular and diastolic pressures is not surprising. Peak CPK cannot be used to predict risk from ventricular fibrillation because all the cases of ventricular fibrillation in this study occurred within the first 24 hours before peak CPKs were available. It can however, to some extent, predict those patients who will go on to develop left ventricular failure, and those at risk $\underset{\mathcal{Q}}{\mathbb{Q}}$ to death in the early hospital convalescent phase $O$ and in the late convalescent phase at home. This 음 is of great importance with regard to rates of ? mobilization after apparently uncomplicated myo- $\vec{F}$ cardial infarction and suggests that patients with $\stackrel{?}{9}$ very high levels of CPK should possibly be mobilized more carefully, and that their post discharge $\frac{\bar{F}}{\bar{S}}$ convalescence should be more gradual and return to work delayed beyond that normally recommended $\mathscr{\complement}$ after uncomplicated infarction. It is particularly $\infty^{\infty}$ interesting to note that three of the four patients $\overrightarrow{0}$ who died at home did not have any complications in hospital and had no other risk factors. Their $\vec{\omega}$ prognosis was considered excellent. Although the figures are too small to draw conclusions and need to be confirmed by larger studies, it is tantalizing to think that such a simple test might identify the patients at risk to late sudden death, and have implications regarding the need for further inves- $N$ tigation, in such individuals with a view to the introduction of preventive therapy.

\section{REFERENCES}

1 Kitchen A H, Pococks S J. Prognosis of patieng with acute myocardial infarction admitted to ${ }_{1}$ coronary care unit. I Survival in Hospital Br Heart 9 1977; 39: 1163-1166.

2 Kitchen A H, Pocock S J. Prognosis of patients wi acute myocardial infarction admitted to a coronat care unit. II Survival after hospital discharge. Br Heart J 1977; 39: 1167-1171.

3 Lundman T, Claes H. Prognostic factors in the Swedish Collaborative coronary care study. Book: Acute and long term management of myocardial ischaemia Astra Conference 1977; 114-124.

4 Bleifeld W et al. Infarct size estimated from serum creatine phosphokinase in relation to LV haemodynamics. Circ 1977; 55: 303-311.

5 NorRIS R M et al. Clinical measurement of myocardial infarct size. Modification of a method for the estimation of total CPK release after MI. Circ 1975; 51: 614-620.

6 SOBEL B E et al. Estimate of infarct size in man and its relation to prognosis. Circ 1972; 46: 640-648.

7 MATHEY $D$ et al. Creatine Kinase release in acute $\mathbb{D}$ myocardial infarction: correlation with clinical, electrocardiographic and pathological findings, $\mathrm{Br}$ Heart $J$ 1975; 45: 1160-1175.

8 DAHL C F et al. Myocardial necrosis from direct current countershock. Circ 1974; 50: 956-961. 